\title{
Bifurcation Analysis of Three-Strategy Imitative Dynamics with Mutations
}

\author{
Wenjun $\mathrm{Hu}^{1,2}$ Haiyan Tian, ${ }^{1}$ and Gang Zhang $\mathbb{C}^{1}$ \\ ${ }^{1}$ College of Mathematics and Information Science, Hebei Normal University, Shijiazhuang 050024, China \\ ${ }^{2}$ Department of Mathematics, Luliang University, Lishi 033000, China \\ Correspondence should be addressed to Gang Zhang; gangzhang@hebtu.edu.cn
}

Received 11 April 2019; Accepted 13 September 2019; Published 30 October 2019

Academic Editor: Constantin Udriste

Copyright (c) 2019 Wenjun Hu et al. This is an open access article distributed under the Creative Commons Attribution License, which permits unrestricted use, distribution, and reproduction in any medium, provided the original work is properly cited.

\begin{abstract}
Evolutionary game dynamics is an important research, which is widely used in many fields such as social networks, biological systems, and cooperative behaviors. This paper focuses on the Hopf bifurcation in imitative dynamics of three strategies (RockPaper-Scissors) with mutations. First, we verify that there is a Hopf bifurcation in the imitative dynamics with no mutation. Then, we find that there is a critical value of mutation such that the system tends to an unstable limit cycle created in a subcritical Hopf bifurcation. Moreover, the Hopf bifurcation exists for other kinds of the considered mutation patterns. Finally, the theoretical results are verified by numerical simulations through Rock-Paper-Scissors game.
\end{abstract}

\section{Introduction}

Evolutionary game dynamics combines game theory and nonlinear dynamics to describe the evolution of the frequencies of strategies in one or more large population $[1,2]$. It has edged into many fields such as networks population [3-6], economics [7, 8], biology [9, 10], management $[11,12]$, and cooperative behaviors [13-16]. There are many important evolutionary game dynamics such as replicator dynamics, imitative dynamics [17], best-response dynamics [18], and so on [19]. The Rock-Paper-Scissors (RPS) [20, 21] is a famous three-strategy game, which describes interactions among three competing species in ecology, sociological systems [22], and theoretical biology [23, 24]. Replicator dynamics is the best-known evolutionary dynamics, which was firstly defined by Taylor and Jonker [25], and has been researched in various fields [26, 27]. In practice, imitative dynamics is a generalized replicator dynamics, which investigates the spreading of strategies in the context of imitation instead of inheritance.

There are some research studies about the imitative dynamics [28-30]. Cheung [28] studied the imitative dynamics for games with continuous strategy space and obtained global convergence and local stability results for imitative dynamics. Wang et al. [29] investigated the imitation dynamics with delay, and they discussed the two-phenotype and threephenotype model and obtained some relevant results for stability. Hu et al. [30] researched the imitative dynamics with discrete delay, and they discovered that the stability would be changed in the discrete delay dynamics and obtained some sufficient conditions. The emphasis of the literatures is the effect of delay in the imitative dynamics. However, the mutation is also a noticeable factor on the study of the stability of the evolutionary dynamics in reality.

Until now, many researchers have studied the effect of mutations in the replicator dynamics [31-34]. Mobilia [31] investigated the oscillatory dynamics in generic RPS games with mutations and found out the existence of the heteroclinic cycles in the RPS model. Nagatani et al. [32] studied a metapopulation model for RPS game with mutation, and they found that the mutation would lead to the phase transitions among three strategies. Toupo et al. [33, 34] researched the effect of mutations in the repeated prisoner's dilemma game and RPS game, and they found that the mutations would result in the Hopf bifurcation in the replicator dynamics. Their research studies illustrate that 
mutation could change the stability of the dynamics, especially lead to bifurcation.

The bifurcation is an important behavior in dynamical systems [35], which has been researched by many scholars [36-39]. Wesson et al. [36, 37] investigated the Hopf bifurcation in two-strategy and three-strategy delayed replicator dynamics, and they demonstrated the existence of Hopf bifurcation and presented an analysis of the limit cycles through Lindstedt's method. Nesrine et al. [38] researched the Hopf bifurcation in RPS game with distributed delays. Umezuki [39] studied the bifurcation of RPS game with discrete-time logit dynamics and showed that some bifurcations would destroy the coexistence of the attractors in the RPS game.

According to the previous literatures, there are few research studies about the bifurcation in imitative dynamics. In this paper, we aim to discuss the Hopf bifurcation in imitative dynamics with mutation. Our research will illustrate that (i) the imitative dynamics appears to be a Hopf bifurcation at the parameter $\gamma$ in the RPS game; (ii) the stability would be changed in the mutative imitation dynamics; and (iii) a subcritical Hopf bifurcation would be exhibited in this dynamics.

The rest of this paper is organized as follows. Section 2 sets the imitation dynamics model without mutation and analyses the stability and bifurcation. Section 3 researches the Hopf bifurcations with mutations in the imitative dynamics. Section 4 gives numerical simulations of the equilibrium and an unstable periodic solution. Section 5 offers concluding remarks.

\section{RPS Model without Mutation}

2.1. Derivation. We consider a symmetric three-phenotype model with pure strategies Rock (R), Scissors (S), and Paper $(\mathrm{P})$ and with payoff matrix:

$$
\begin{aligned}
& R \\
& S
\end{aligned}\left(\begin{array}{ccc}
1 & S & P \\
0 & 1+\gamma & 0 \\
1+\gamma & 0 & 1
\end{array}\right), \quad \gamma>0 .
$$

The payoff matrix means that each strategy gets a payoff 1 when playing against itself, and the loser gets a payoff 0 while the winner gets $1+\gamma$. Let $\left(x_{1}, x_{2}, x_{3}\right)$ denote the frequency of $(\mathrm{R}, \mathrm{S}, \mathrm{P})$ and $\left(f_{1}, f_{2}, f_{3}\right)$ the expected payoff of $(\mathrm{R}, \mathrm{S}, \mathrm{P})$ with $f_{i}(x)=\sum_{j=1}^{3} x_{j} a_{i j}$, where $x=\left(x_{1}, x_{2}, x_{3}\right)$ with $\sum_{i=1}^{3} x_{i}=1$ and $a_{i j}$ denotes the payoff of $S_{i}$-individual plays against a $S_{j}$ individual in which $i, j=1,2,3$.

The classic imitation dynamics tacitly supposes that an individual is randomly selected from the population and awarded the same opportunity to change the strategy. That is, when an individual using $S_{i}$ plays against an individual using $S_{j}$, the imitation rate that the $S_{j}$ strategist switches to $S_{i}$ is denoted by $F_{i j}$ for $i, j=1,2,3$. In the previous literature, it is assumed that the imitation rate $F_{i j}$ depends on the expected payoffs $f_{i}(x)$ and $f_{j}(x)$ :

$$
F_{i j}(x)=F\left(f_{i}(x), f_{j}(x)\right), \quad(i, j=1,2,3),
$$

where the function $F(u, v)$ defines the imitation rule. Here, we take $F(u, v)=u /(u+v)$, i.e.,

$$
F_{i j}(x(t))=\frac{f_{i}(x(t))}{f_{i}(x(t))+f_{j}(x(t))}, \quad(i, j=1,2,3)
$$

For ease of notation, write $\left(x_{1}, x_{2}, x_{3}\right)=(x, y, z)$. Under this condition, the imitation dynamics equations can be written as follows:

$$
\left\{\begin{array}{l}
\dot{x}=x\left(\frac{f_{1}-f_{2}}{f_{1}+f_{2}} y+\frac{f_{1}-f_{3}}{f_{1}+f_{3}} z\right), \\
\dot{y}=y\left(\frac{f_{2}-f_{1}}{f_{2}+f_{1}} x+\frac{f_{2}-f_{3}}{f_{2}+f_{3}} z\right), \\
\dot{z}=z\left(\frac{f_{3}-f_{1}}{f_{3}+f_{1}} x+\frac{f_{3}-f_{2}}{f_{3}+f_{2}} y\right) .
\end{array}\right.
$$

Since $x, y$, and $z$ are the frequencies of the three strategies, the region of interest is the three-dimensional simplex in $R^{3}$ :

$$
\sum \equiv\left\{(x, y, z) \in R^{3}: x+y+z=1, \quad(x, y, z \geq 0)\right\} .
$$

So, we can eliminate $z$ using $z=1-x-y$ and the projection of $\sum$ into the $x-y$ plane: $S \equiv\left\{(x, y) \in R^{2}\right.$ : $\left.(x, y, 1-x-y) \in \sum\right\}$. In this case, equation (4) can be written as

$$
\begin{aligned}
\dot{x}= & x\left[y \frac{(2+\gamma) x+(1+2 \gamma) y-(1+\gamma)}{-\gamma x+(1+\gamma) y+(1+\gamma)}\right. \\
& \left.+(1-x-y) \frac{(1-\gamma) x+(2+\gamma) y-1}{(1+\gamma) x+\gamma y+1}\right], \\
\dot{y}= & y\left[-x \frac{(2+\gamma) x+(1+2 \gamma) y-(1+\gamma)}{-\gamma x+(1+\gamma) y+(1+\gamma)}\right. \\
& \left.+(1-x-y) \frac{-x-(1+\gamma) y+2+\gamma}{-(1+\gamma) x-\gamma y+\gamma}\right] .
\end{aligned}
$$

2.2. Stability of Equilibria. System (6) has four equilibria:

$$
\begin{aligned}
& e_{1}=(0,0), \\
& e_{2}=(0,1), \\
& e_{3}=(1,0), \\
& x^{*}=\left(\frac{1}{3}, \frac{1}{3}\right) .
\end{aligned}
$$

In order to discuss the stability of these equilibria, we linearize equation (6). As a result, we can analyze the stability of each point through the eigenvalues of the Jacobian. The eigenvalues of the three corner equilibria can be calculated as shown in Table 1.

From above analysis, in the nonmutation RPS equation, each corner of $S$ is a saddle point. 
TABle 1: The eigenvalues of equilibria.

\begin{tabular}{lc}
\hline Points & Eigenvalues \\
\hline$(1,0)$ & $\lambda_{1}=-1, \lambda_{2}=\gamma / \gamma+2$ \\
$(0,1)$ & $\lambda_{2}=\gamma / \gamma+2, \lambda_{1}=-1$ \\
$(0,0)$ & $\lambda_{1}=-1, \lambda_{2}=\gamma / \gamma+2$ \\
$(1 / 3,1 / 3)$ & $\lambda_{1,2}=1-\gamma / 4(\gamma+2) \pm(\sqrt{3}(1+\gamma) / 4(\gamma+2)) i$ \\
\hline
\end{tabular}

Next, we consider an important equilibrium $x^{*}$; first, we discuss the stability in the nonmutation system. Since there are two imaginary eigenvalues at this equilibrium point, we think there might be a Hopf bifurcation at $x^{*}$.

2.3. Hopf Bifurcation. First, we introduce a lemma about Hopf bifurcation in the vector field.

Lemma 1 (see [40]). Suppose that system

$$
\dot{x}=f_{\mu}(x), \quad x \in \mathbb{R}^{n}, \mu \in \mathbb{R},
$$

has an equilibrium $\left(x_{0}, \mu_{0}\right)$ at which the following properties are satisfied:

(H1) $D_{x} f_{\mu_{0}}\left(x_{0}\right)$ has a simple pair of pure imaginary eigenvalues and no other eigenvalues with zero real parts

(H2) $d=d /\left.d \mu(\operatorname{Re} \lambda(\mu))\right|_{\mu=\mu_{0}} \neq 0$

(H3) $a=a\left(\mu_{0}\right) \neq 0$, where $a\left(\mu_{0}\right)$ is the first Lyapunov coefficient

Then, the system undergoes a Hopf bifurcation at $\mu=\mu_{0}$.

The coefficient $a(\mu)$ can be calculated as follows. On the center manifold, $f_{\mu}(x)$ has the following form near the origin:

$$
\left(\begin{array}{c}
\dot{x} \\
\dot{y}
\end{array}\right)=\left(\begin{array}{cc}
\operatorname{Re} \lambda(\mu) & -\operatorname{Im} \lambda(\mu) \\
\operatorname{Im} \lambda(\mu) & \operatorname{Re} \lambda(\mu)
\end{array}\right)\left(\begin{array}{l}
x \\
y
\end{array}\right)+\left(\begin{array}{c}
f^{1}(x, y, \mu) \\
f^{2}(x, y, \mu)
\end{array}\right),
$$

where $f^{1}$ and $f^{2}$ are nonlinear functions in $x$ and $y$ and $\lambda(\mu)$ and $\bar{\lambda}(\mu)$ are the eigenvalues of the linearized system around the equilibrium at the origin. Especially, at the bifurcation point (i.e., $\mu=0, \lambda_{1,2}= \pm i \omega$ ), the coefficient is given by

$$
\begin{aligned}
a(\mu)= & \frac{f_{x x x}^{1}+f_{x y y}^{1}+f_{x x y}^{2}+f_{y y y}^{2}}{16} \\
& +\frac{f_{x y}^{1}\left(f_{x x}^{1}+f_{y y}^{1}\right)-f_{x y}^{2}\left(f_{x x}^{2}+f_{y y}^{2}\right)}{16 \omega} \\
& +\frac{f_{y y}^{1} f_{y y}^{2}-f_{x x}^{1} f_{x x}^{2}}{16 \omega} .
\end{aligned}
$$

Lemma 2 (see [41]). Consider the system form (8); for sufficiently small $\mu$, the following four cases hold: (i) $d>0, a>0$ : unstable equilibrium for $\mu>0$ and asymptotically stable equilibrium for $\mu<0$, with unstable periodic orbit (i.e., subcritical) for $\mu<0$

(ii) $d>0, a<0$ : unstable equilibrium for $\mu>0$ and asymptotically stable equilibrium for $\mu<0$, with asymptotically stable periodic orbit (i.e., supercritical) for $\mu>0$

(iii) $d<0, a>0$ : unstable equilibrium for $\mu<0$ and asymptotically stable equilibrium for $\mu>0$, with unstable periodic orbit (i.e., subcritical) for $\mu>0$

(iv) $d<0, a<0$ : unstable equilibrium for $\mu>0$ and asymptotically stable equilibrium for $\mu<0$, with asymptotically stable periodic orbit (i.e., supercritical) for $\mu<0$

Next, we give a theorem to illustrate the bifurcation in dynamics (6).

Theorem 1. The imitation dynamics (6) exhibits a subcritical Hopf bifurcation at $\gamma=1$. Moreover, when $\gamma>1$, the interior equilibrium is locally stable, and it is unstable when $\gamma<1$.

Proof. (i) When $\gamma \neq 1$, the sign of the real part of eigenvalues can be determined through Table 1 . That is,

$\gamma>1, \operatorname{Re}(\lambda)=\frac{1-\gamma}{4(\gamma+2)}<0, \quad$ (stability),

$\gamma<1, \operatorname{Re}(\lambda)=\frac{1-\gamma}{4(\gamma+2)}>0, \quad$ (instability).

(ii) When $\gamma=1$, as the formula in Lemma 1, we obtain the nonlinear function form,

$$
\begin{aligned}
f^{1}(x, y)= & x\left[(1-x-y) \frac{3 y-1}{2 x+y+1}+y \frac{3 x+3 y-2}{-x+2 y+2}\right] \\
& +\frac{\sqrt{3}}{6} y, \\
f^{2}(x, y)= & y\left[(1-x-y) \frac{-x-2 y+3}{-2 x-y+1}-x \frac{3 x+3 y-2}{-x+2 y+2}\right] \\
& -\frac{\sqrt{3}}{6} x .
\end{aligned}
$$

We can obtain the Lyapunov coefficient through Matlab as follows:

$$
\begin{aligned}
& a(\gamma)=\left.\frac{27\left(\gamma^{3}+4 \gamma^{2}+2 \gamma-3\right)}{16(\gamma+2)^{3}}\right|_{\gamma=1}=\frac{1}{4}>0 . \\
& d(\gamma)=\frac{d}{d(\gamma)}(\operatorname{Re} \lambda(\gamma))=-\left.\frac{3}{4(\gamma+2)^{2}}\right|_{\gamma=1}<0 .
\end{aligned}
$$




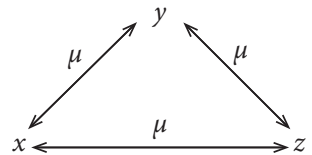

FIgURE 1: The global mutation in RPS.

According to Lemma 2, the Hopf bifurcation is subcritical at $\gamma=1$.

\section{RPS Model with Mutations}

In this section, we examine the imitative dynamics with all kinds of mutations, including global mutations, single mutation, double mutations, and so on.

3.1. Global Mutations in RPS Model. First, we discuss the global mutations in imitative dynamics. The relationship of mutations is shown in Figure 1.

In this case, the dynamics becomes the following form with mutant coefficient $\mu(\mu \geq 0)$ :

$$
\left\{\begin{array}{c}
\dot{x}=x\left[(1-x-y) \frac{(1-\gamma) x+(2+\gamma) y-1}{(1+\gamma) x+\gamma y+1}\right. \\
\left.+y \frac{(2+\gamma) x+(1+2 \gamma) y-(1+\gamma)}{-\gamma x+(1+\gamma) y+(1+\gamma)}\right]+\mu(1-3 x), \\
\dot{y}=y\left[(1-x-y) \frac{-x-(1+\gamma) y+2+\gamma}{-(1+\gamma) x-\gamma y+\gamma}\right. \\
\left.-x \frac{(2+\gamma) x+(1+2 \gamma) y-(1+\gamma)}{-\gamma x+(1+\gamma) y+(1+\gamma)}\right]+\mu(1-3 y) .
\end{array}\right.
$$

We give a theorem to illustrate the bifurcation in dynamics (14) as follows.

Theorem 2. The following two conclusions are established for imitative dynamics (14):

(i) There exists a subcritical Hopf bifurcation at $\mu=1-$ $\gamma / 12(\gamma+2) \quad$ when $\quad \gamma<1$. Moreover, for $\mu>1-\gamma / 12(\gamma+2)$, the interior equilibrium is locally stable, and for $\mu<1-\gamma / 12(\gamma+2)$, it is unstable

(ii) The interior equilibrium is locally stable when $\gamma \geq 1$

Proof. The Jacobian matrix of dynamics $(14)$ at $(1 / 3,1 / 3)$ is

$$
J\left(\frac{1}{3}, \frac{1}{3}\right)=\left[\begin{array}{cc}
\frac{1-\gamma}{6(\gamma+2)}-3 \mu & \frac{2 \gamma+1}{6(\gamma+2)} \\
-\frac{1}{6} & \frac{1-\gamma}{6(\gamma+2)}-3 \mu
\end{array}\right] \text {, }
$$

and the conjugate complex eigenvalues are

$$
\lambda_{1,2}(\mu)=\frac{1-\gamma}{4(\gamma+2)}-3 \mu \pm \frac{\sqrt{3}(\gamma+1)}{4(\gamma+2)} i
$$

Let $\operatorname{Re}\left(\lambda_{1,2}\right)=0$, then $\mu=1-\gamma / 12(\gamma+2)$. One can obtain the following:

(i) $\gamma<1$ : if $\mu=1-\gamma / 12(\gamma+2)$, then the Jacobian matrix has a pair of pure complex eigenvalues

(ii) $\gamma \geq 1: \operatorname{Re}(\lambda)<0$ is always correct, i.e., the interior equilibrium is locally stable

Similar to the proof in Theorem 1, the nonlinear functions can be obtained as follows:

$$
\left\{\begin{array}{l}
f^{1}(x, y)=x\left[y \frac{(2+\gamma) x+(1+2 \gamma) y-(1+\gamma)}{-\gamma x+(1+\gamma) y+(1+\gamma)}\right. \\
\left.+(1-x-y) \frac{(1-\gamma) x+(2+\gamma) y-1}{(1+\gamma) x+\gamma y+1}\right] \\
+\frac{1-\gamma}{12(\gamma+2)}(1-3 x)+\frac{\sqrt{3}(\gamma+1)}{4(\gamma+2)} y, \\
f^{2}(x, y)=y\left[x \frac{(1+\gamma)-(2+\gamma) x+(1+2 \gamma) y}{-\gamma x+(1+\gamma) y+(1+\gamma)}\right. \\
\left.+(1-x-y) \frac{-x-(1+\gamma) y+2+\gamma}{-(1+\gamma) x-\gamma y+\gamma}\right] \\
+\frac{1-\gamma}{12(\gamma+2)}(1-3 y)-\frac{\sqrt{3}(\gamma+1)}{4(\gamma+2)} x .
\end{array}\right.
$$

The Lyapunov coefficient can be calculated as follows:

$$
\begin{aligned}
a(\gamma) & =\frac{27\left(\gamma^{3}+4 \gamma^{2}+2 \gamma-3\right)}{16(\gamma+2)^{3}}>0, \quad(\text { for } 0<\gamma<1) \\
d & =\frac{d}{d(\mu)}(\operatorname{Re} \lambda(\mu))=\frac{d}{d(\mu)}\left(\frac{1-\gamma}{4(\gamma+2)}-3 \mu\right)=-3<0 .
\end{aligned}
$$

According to Lemma 2, the Hopf bifurcation is subcritical at $\mu=1-\gamma / 12(\gamma+2)$.

The results in Theorem 1 and Theorem 2 show that the situation in dynamics (8) is different from dynamics (14). While the interior equilibrium is always unstable when $\gamma<1$ in the former, the interior equilibrium is locally stable for $\mu>1-\gamma / 12(\gamma+2)$ when $\gamma<1$ in the latter.

3.2. Other Mutations in RPS Model. In this section, we discuss the other mutations in imitative dynamics; the situation becomes complex as one adds more mutant pathways. For the ease of research, let us restrict attention to mutant forms that ensure $(x, y)=(1 / 3,1 / 3)$ as the inner equilibrium for all values of $\gamma$ and $\mu$. 


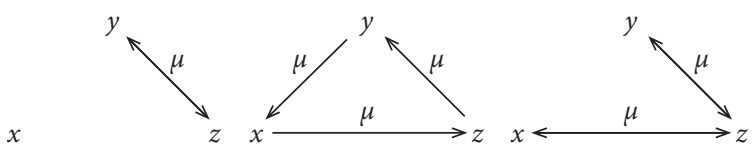

FIGURE 2: Three representative mutations in RPS.

TABLE 2: Hopf curve of different mutant forms.

\begin{tabular}{lrr}
\hline Forms & Numbers & Hopf curve \\
\hline$y \rightleftarrows z$ & 2 & $\mu_{c}=1-\gamma / 4(\gamma+2)$ \\
$x \longrightarrow y \longrightarrow z \longrightarrow x$ & 3 & $\mu_{c}=1-\gamma / 6(\gamma+2)$ \\
$x \leftrightarrows z, z \rightleftarrows y$ & 4 & $\mu_{c}=1-\gamma / 8(\gamma+2)$ \\
\hline
\end{tabular}

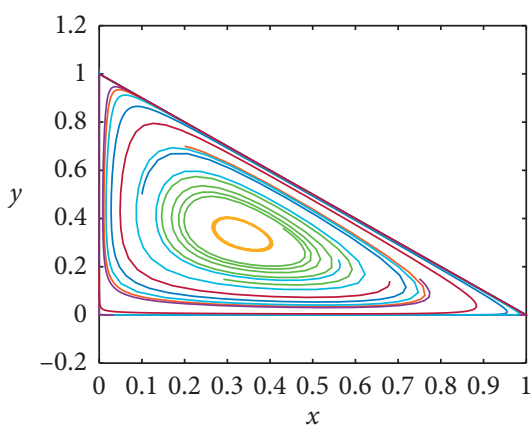

(a)

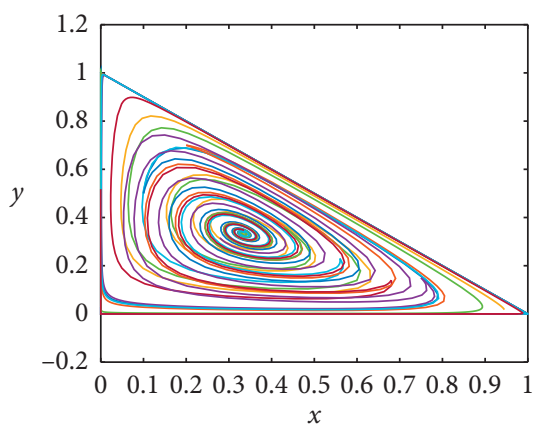

(b)

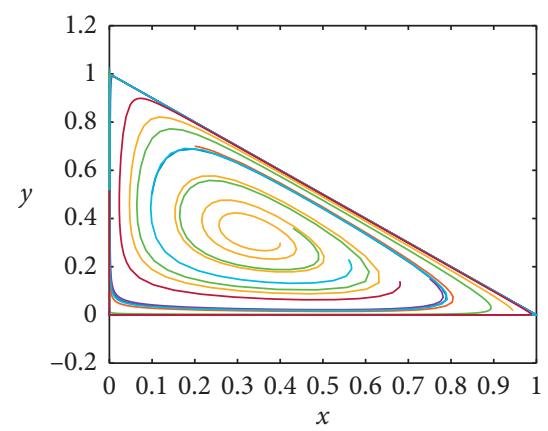

(c)

Figure 3: The interior equilibrium's changing situation with different $\gamma$. (a) When $\gamma=1$, the limit cycle occurs around the interior equilibrium. (b) When $\gamma=1.5$, the interior equilibrium is stable. (c) When $\gamma=0.8$, the interior equilibrium is unstable.

Here, we discuss the following three kinds of mutations in RPS imitative dynamics (see Figure 2): (i) single mutation between two strategies; (ii) single-cycle mutations among three strategies; and (iii) double mutations between two strategies. These mutant forms are shown in Table 2. As the cycle symmetry of the RPS game, it suffices to consider one of the three possible single mutation and double mutations. In this case, we just consider the following representative mutations.

For the imitative dynamics with these three kinds of mutations, similar subcritical Hopf bifurcation at $\mu=\mu_{c}$ would be present. This is different from the stability in nonmutation imitative dynamics, which is always unstable when $\gamma<1$.

\section{Numerical Simulations}

In this section, we propose to compare the properties of the bifurcating periodic solution. Here, we report two simulation results for imitative dynamics with nonmutation and mutation, respectively.

Example 1. In the imitative dynamics (6), we take

$$
\begin{aligned}
& \gamma=1.5, \\
& \gamma=1, \\
& \gamma=0.8,
\end{aligned}
$$

into the equation. Through the Matlab software, one can obtain the following results (see Figure 3).

In Figure 3, the numerical simulation shows that the interior equilibrium $x^{*}$ is asymptotically stable when $\gamma>1$ (i.e., $\gamma=1.5$ ). However, when $\gamma=1$, the system state tends to a unstable periodic solution, and when $\gamma<1$ (i.e., $\gamma=0.8$ ), the interior equilibrium $x^{*}$ is unstable.

Example 2. In the imitative dynamics (14), let

$$
\begin{aligned}
\gamma & =1.5, \\
\mu & =0.02, \\
\mu & =0.002, \\
\gamma & =0.8, \\
\mu & =0.02, \\
\mu & =0.002, \\
\mu & =0.00595 .
\end{aligned}
$$

Through the Matlab software, one can obtain the following results (see Figures 4 and 5).

In Figure 4, the numerical simulation shows that the interior equilibrium $x^{*}$ is asymptotically stable when $\gamma=1.5$ (i.e., $\gamma>1$ ) for any value of $\mu$, such as $\mu=0.02$ and $\mu=0.002$.

In Figure 5, the numerical simulation shows the following: (i) the Hopf curve in dynamics (14), i.e., the 


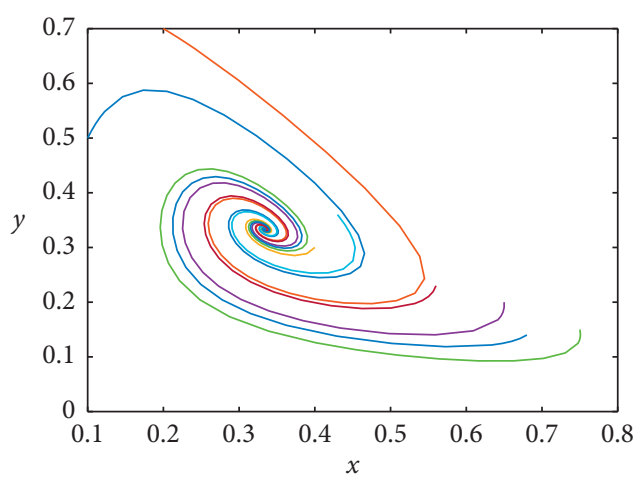

(a)

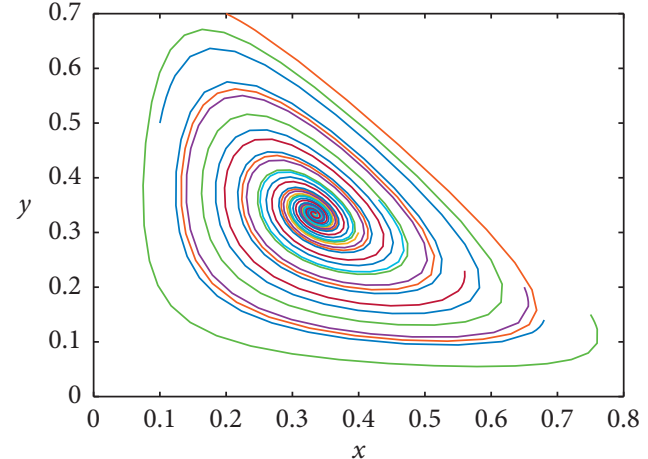

(b)

Figure 4: The interior equilibrium's changing situation with the same $\gamma$ and different $\mu$. (a) $\gamma=1.5$ and $\mu=0.02$. (b) $\gamma=1.5$ and $\mu=0.002$.

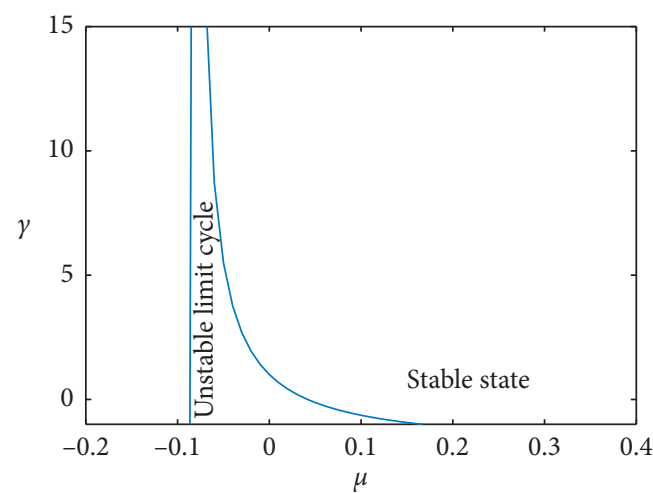

(a)

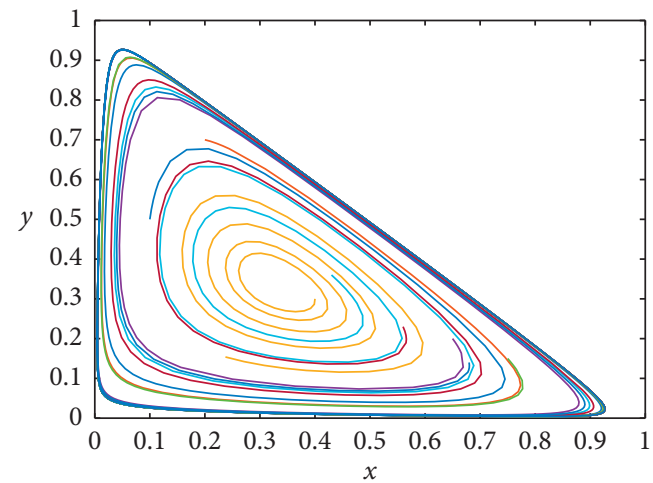

(c)

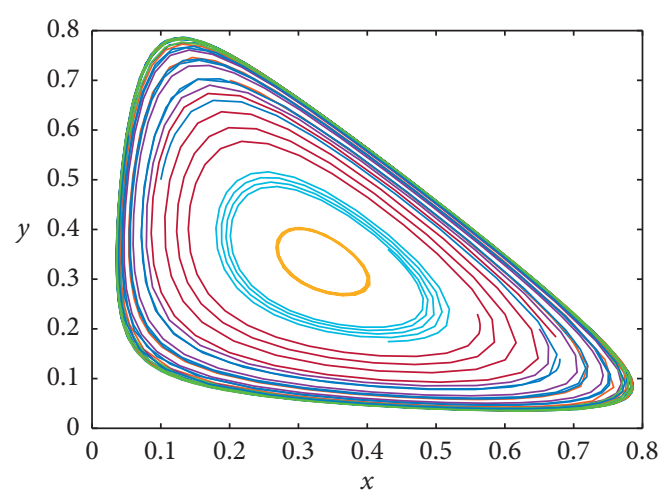

(b)

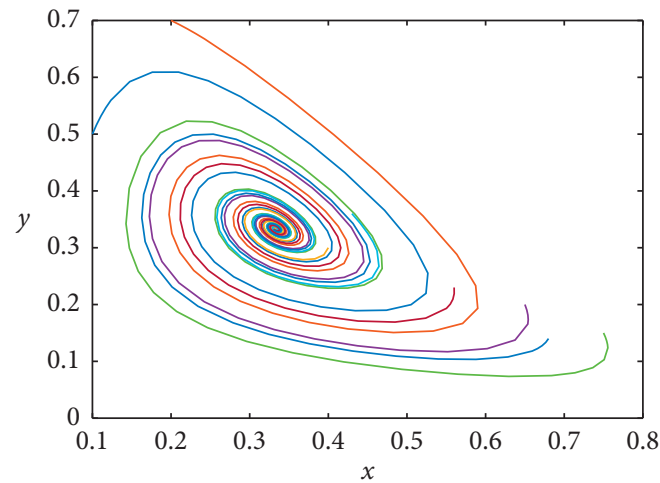

(d)

Figure 5: (a) The stability and limit cycle with different $\gamma$ and $\mu$ (Hopf curve). (b) When $\gamma=0.8$ and $\mu=0.00595$, the limit cycle occurs around $x^{*}$. (c) When $\gamma=0.8$ and $\mu=0.002$, the interior equilibrium is unstable. (d) When $\gamma=0.8$ and $\mu=0.02$, the interior equilibrium is stable.

criticality of $\mu$, changes with $\gamma$; (ii) the interior equilibrium $x^{*}$ would be stable when $\gamma=0.8$ (i.e., $\gamma<1$ ); and (iii) when $\mu=0.00595$, the system state tends to a unstable periodic solution, and when $\mu>0.00595$, the interior equilibrium $x^{*}$ is stable and is unstable when $\mu<0.00595$. The marks in Figures 4 and 5 are same as the description in Figure 3.

\section{Conclusion}

In this paper, the stability of the interior equilibrium has been mainly investigated for imitative dynamics with mutations. Different from the result in replicator dynamics $[31,34]$, the stability of the interior equilibrium has been 
changed with the mutations, and a subcritical Hopf bifurcation appears.

For the imitative dynamics in the RPS game, the stability is changed at the parameter $\gamma$ in the payoff matrix with no mutation, and the mutation $\mu$ in global mutation. In the dynamics with no mutation, the interior equilibrium is locally stable when $\gamma>1$ and is unstable when $\gamma<1$, and a subcritical Hopf bifurcation appears at $\gamma=1$ in given payoff matrix. In the imitative dynamics with global mutation, the interior equilibrium is stable when $\gamma \geq 1$, and it is different from the case when $\gamma<1$. There is a subcritical Hopf bifurcation at $\mu=1-\gamma / 12(\gamma+2)$, and the interior equilibrium is locally stable when $\mu>1-\gamma / 12(\gamma+2)$ and is unstable when $\mu<1-\gamma / 12(\gamma+2)$.

If we change the number of parameters (i.e., from one to two), the stability and bifurcation would become much more complicated. Furthermore, some numerical examples have been given to illustrate the effectiveness of our results. As an extension to this work, we plan to discuss the imitation dynamics with delays and mutations.

\section{Data Availability}

No data were used to support this study.

\section{Conflicts of Interest}

The authors declare that they have no conflicts of interest.

\section{Authors' Contributions}

All authors contributed equally and significantly in writing this paper and read and approved the final manuscript.

\section{Acknowledgments}

The authors gratefully acknowledge the support of the National Natural Science Foundation of China (NNSF) through grant no. 11562006, the Innovation Foundation for Graduate in Hebei Normal University (no. YJBS2019001), the Scientific and Technological Innovation Programs of Higher Education Institutions in Shanxi Province (no. 2019L0940), the Science Foundation of Hebei Normal University (no. L2018B01), and the Natural Science Foundation of Shanxi Province (no. 201801D121009).

\section{References}

[1] J. M. Smith, Evolution and the Theory of Games, Cambridge University Press, Cambridge, UK, 1982.

[2] J. Hofbauer and K. Sigmund, Evolutionary Games and Population Dynamics, Cambridge University Press, Cambridge, UK, 1998.

[3] M. R. D’Orsogna and M. Perc, "Statistical physics of crime: a review," Physics of Life Reviews, vol. 12, pp. 1-21, 2015.

[4] D. Madeo and C. Mocenni, "Game interactions and dynamics on networked populations," IEEE Transactions on Automatic Control, vol. 60, no. 7, pp. 1801-1810, 2015.

[5] Z. Wang, M. A. Andrews, Z.-X. Wu, L. Wang, and C. T. Bauch, "Coupled disease-behavior dynamics on complex networks: a review," Physics of Life Reviews, vol. 15, pp. 1-29, 2015.

[6] J. M. Pacheco, A. Traulsen, and M. A. Nowak, "Coevolution of strategy and structure in complex networks with dynamical linking," Physical Review Letters, vol. 97, no. 25, Article ID 258103, 2006.

[7] H. Xu, C. Tian, X. Xiao, and S. Fan, "Evolutionary investors' power-based game on networks," Applied Mathematics and Computation, vol. 330, pp. 125-133, 2018.

[8] H. Xu, S. Fan, C. Tian, and X. Xiao, "Evolutionary investor sharing game on networks," Applied Mathematics and Computation, vol. 340, pp. 138-145, 2019.

[9] R. Liu and G. Liu, "Asymptotic behavior of a stochastic TwoSpecies competition model under the effect of disease," vol. 2018, Article ID 3127404, 15 pages, 2018.

[10] J. Yang and X. Wang, "Threshold dynamics of an SIR model with nonlinear incidence rate and age-dependent susceptibility," vol. 2018, Article ID 9613807, 15 pages, 2018.

[11] R. Lu, X. Wang, H. Yu, and D. Li, "Multiparty evolutionary game model in coal mine safety management and its application," Complexity, vol. 2018, Article ID 9620142, 10 pages, 2018.

[12] X. Wang, R. Lu, H. Yu, and D. Li, "Stability of the evolutionary game system and control strategies of behavior instability in coal mine safety management," Complexity, vol. 2019, Article ID 6987427, 14 pages, 2019.

[13] H.-X. Yang and X. Chen, "Promoting cooperation by punishing minority," Applied Mathematics and Computation, vol. 316, pp. 460-466, 2018.

[14] J. Gómez-Gardeñes, M. Campillo, L. M. Floría, and Y. Moreno, "Dynamical organization of cooperation in complex topologies," Physical Review Letters, vol. 98, no. 10, Article ID 108103, 2007.

[15] M. A. Nowak, A. Sasaki, C. Taylor, and D. Fudenberg, "Emergence of cooperation and evolutionary stability in finite populations," Nature, vol. 428, no. 6983, pp. 646-650, 2004.

[16] M. A. Nowak, "Five rules for the evolution of cooperation," Science, vol. 314, no. 5805, pp. 1560-1563, 2006.

[17] D. Vilone, J. J. Ramasco, Angel Sánchez, and M. S. Miguel, "Social and strategic imitation: the way to consensus," Science Reports, vol. 2, Article ID 686, 2012.

[18] C. H. Hommes and M. I. Ochea, "Multiple equilibria and limit cycles in evolutionary games with Logit Dynamics," Games and Economic Behavior, vol. 74, no. 1, pp. 434-441, 2012.

[19] R. Cressman and Y. Tao, "The replicator equation and other game dynamics," Proceedings of the National Academy of Sciences, vol. 111, no. S3, pp. 10810-10817, 2014.

[20] B. Sinervo and C. M. Lively, "The rock-paper-scissors game and the evolution of alternative male strategies," Nature, vol. 380, no. 6571, pp. 240-243, 1996.

[21] B. Xu, H. Zhou, and Z. Wang, "Cycle frequency in standard Rock-Paper-Scissors games: evidence from experimental economics," Physica A: Statistical Mechanics and Its Applications, vol. 392, no. 20, pp. 4997-5005, 2013.

[22] T. Platkowski and J. Zakrzewski, "Asymptotically stable equilibrium and limit cycles in the RPS game in a population of players with complex personalities," Physica A: Statistical Mechanics and Its Applications, vol. 390, no. 23-24, pp. 4219-4226, 2011.

[23] M. A. Nowak and K. Sigmund, "Evolutionary dynamics of biological games,” Science, vol. 303, no. 5659, pp. 793-799, 2004.

[24] E. Kukla and T. Platkowski, "Onset of limit cycles in population games with attractiveness driven strategy choice," Chaos, Soliton and Fractals, vol. 56, pp. 77-82, 2013. 
[25] P. D. Taylor and L. B. Jonker, "Evolutionary stable strategies and game dynamics," Mathematical Biosciences, vol. 40, no. 1-2, pp. 145-156, 1978.

[26] Y. Tao and Z. Wang, "Effect of time delay and evolutionarily stable strategy," Journal of Theoretical Biology, vol. 187, no. 1, pp. 111-116, 1997.

[27] J. S. Weitz, C. Eksin, K. Paarporn, S. P. Brown, and W. C. Ratcliff, "An oscillating tragedy of the commons in replicator dynamics with game environment feedback," Proceedings of the National Academy of Sciences of the United Atates of America, vol. 113, no. 47, pp. E7518-E7525, 2016.

[28] M.-W. Cheung, "Imitative dynamics for games with continuous strategy space," Games and Economic Behavior, vol. 99, pp. 206-223, 2016.

[29] S.-C. Wang, J.-R. Yu, S. Kurokawa, and Y. Tao, "Imitation dynamics with time delay," Journal of Theoretical Biology, vol. 420 , pp. 8-11, 2017.

[30] W. Hu, G. Zhang, and H. Tian, "The stability of imitation dynamics with discrete distributed delays," Physica A: Statistical Mechanics and Its Applications, vol. 521, pp. 218-224, 2019.

[31] M. Mobilia, "Oscillatory dynamics in rock-paper-scissors games with mutations," Journal of Theoretical Biology, vol. 264, no. 1, pp. 1-10, 2010.

[32] T. Nagatani, G. Ichinose, and K.-I. Tainaka, "Metapopulation model for rock-paper-scissors game: mutation affects paradoxical impacts," Journal of Theoretical Biology, vol. 450, pp. 22-29, 2018.

[33] D. F. P. Toupo, D. G. Rand, and S. H. Strogatz, "Limit cycles sparked by mutation in the repeated prisoner's Dilemma," International Jounal of Bifurcation and Chaos, vol. 24, no. 12, Article ID 1430035, 2014.

[34] D. F. P. Toupo and S. H. Strogatz, "Nonlinear dynamics of the rock-paper-scissors game with mutations," Physical Review E, vol. 91, no. 5, Article ID 052907, 2015.

[35] J. N. Webb, Game Theory Decisions, Interaction and Evolution, Springer Undergraduate Mathematics Series, New York, NY, USA, 2006.

[36] E. Wesson and R. Rand, "Hopf bifurcations in delayed rockpaper-scissors replicator dynamics," Dynamic Games and Applications, vol. 6, no. 1, pp. 139-156, 2016.

[37] E. Wessonn, R. Rand, and David Rand, "Hopf bifurcations in two-strategy delayed replicator dynamics," International Jounal of Bifurcation and Chaos, vol. 26, no. 1, Article ID 1650006, 2016.

[38] B. K. Nesrine, E. A. Rachid, and H. Yezekael, "Hopf bifurcations in replicator dynamics with distributed delays," 2017, https:// arxiv.org/abs/1703.06721.

[39] Y. Umezuki, "Bifurcation analysis of the rock-paper-scissors game with discrete-time logit dynamics," Mathematical Social Sciences, vol. 95, pp. 54-65, 2018.

[40] J. Guckenheimer and P. Holmes, Nonlinear Oscillations, Dynamical Systems, and Bifurcations of Vector Fields, Springer, New York, NY, USA, 2002.

[41] S. Wiggins, Intoduction to Applied Nonlinear Dynamical Systems and Chaos, Springer, New York, NY, USA, 2003. 


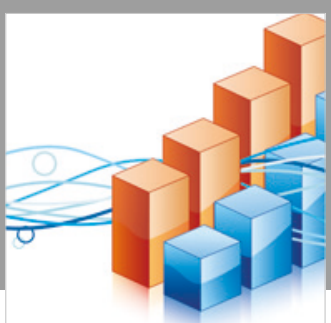

Advances in

Operations Research

\section{-n-m}
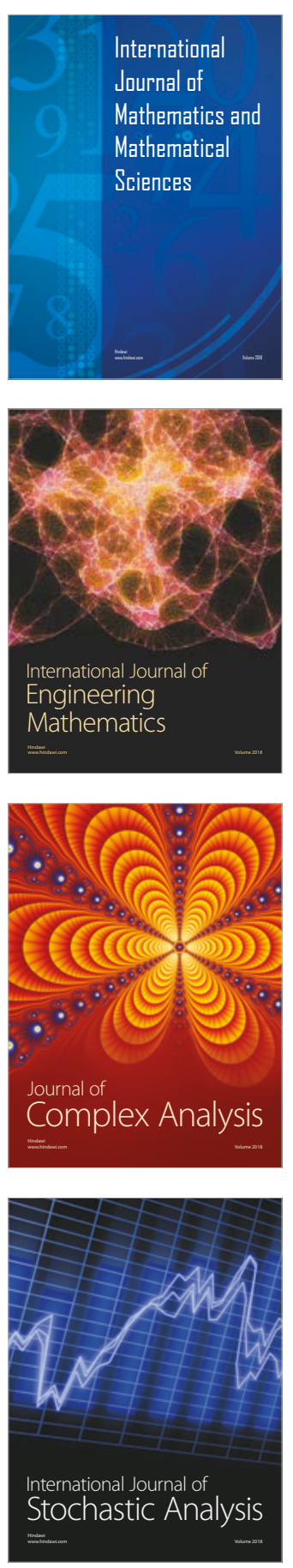
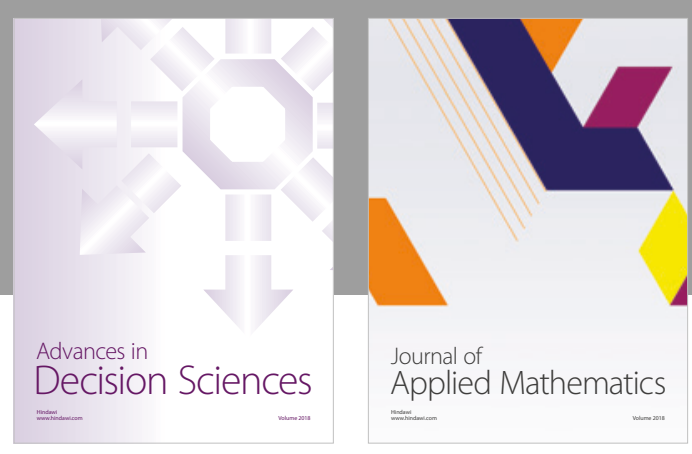

Journal of

Applied Mathematics
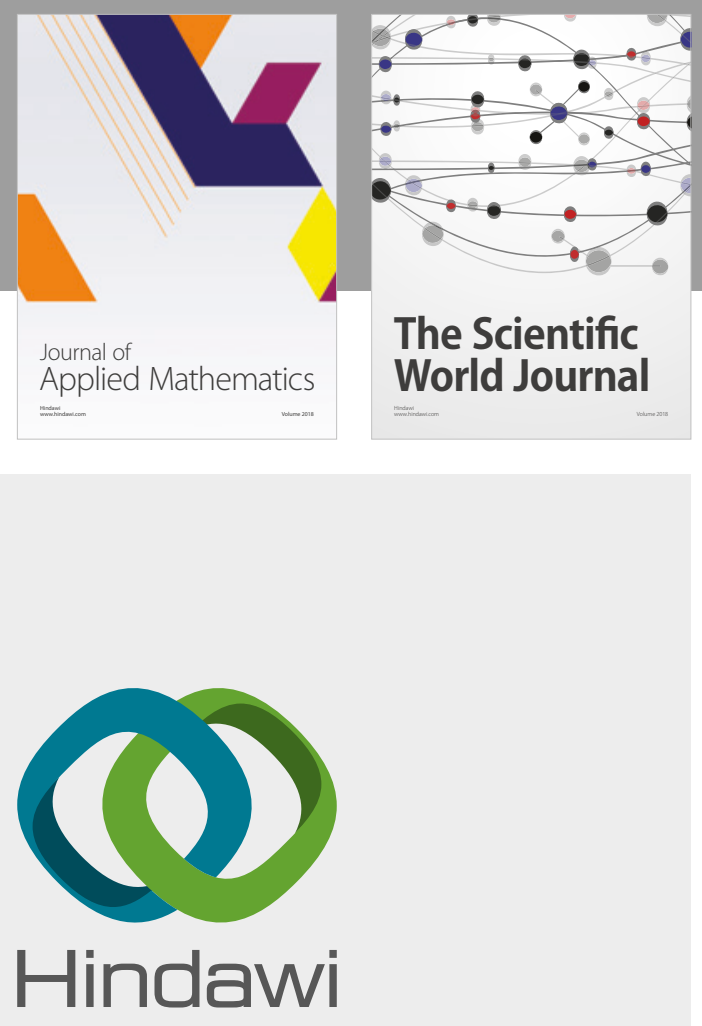

Submit your manuscripts at

www.hindawi.com

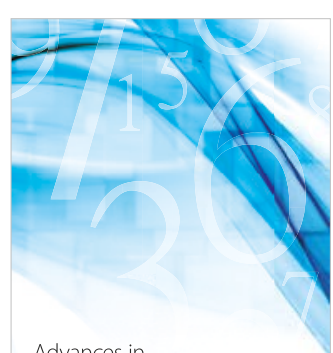

Advances in
Numerical Analysis
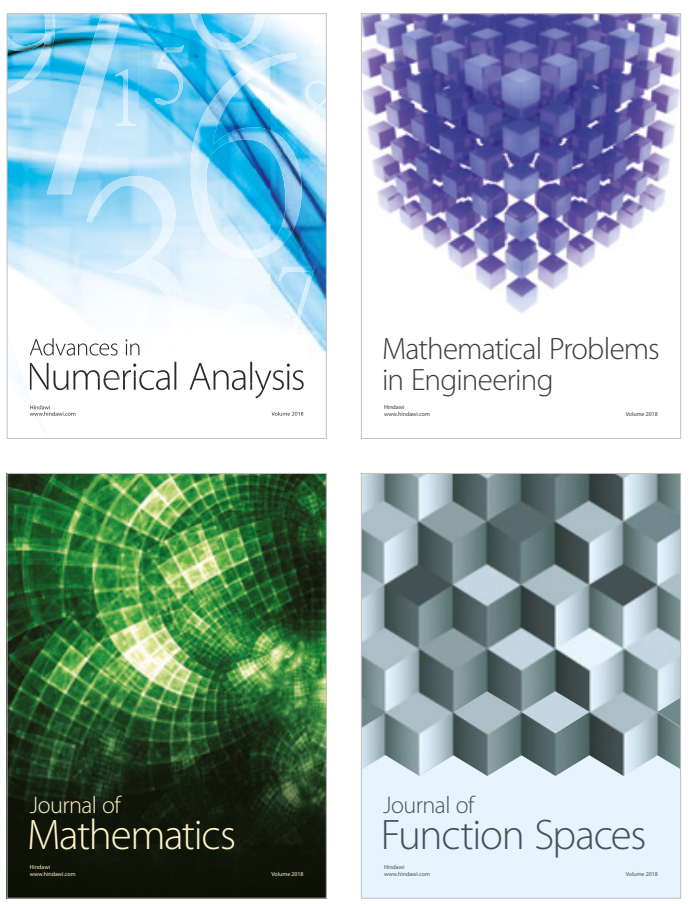

Mathematical Problems in Engineering

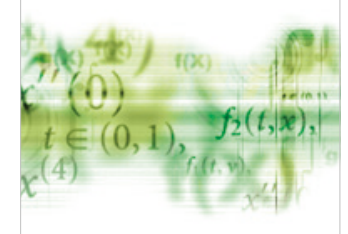

International Journal of

Differential Equations

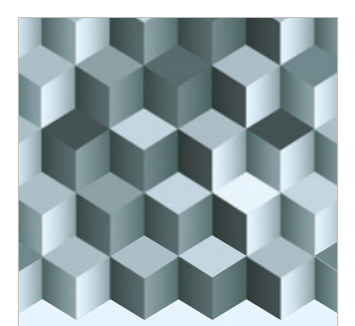

Journal of

Function Spaces
The Scientific

World Journal

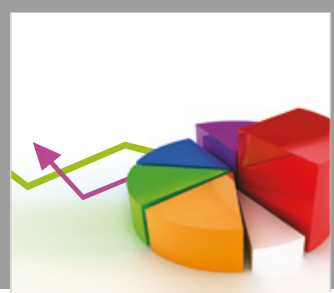

Journal of

Probability and Statistics
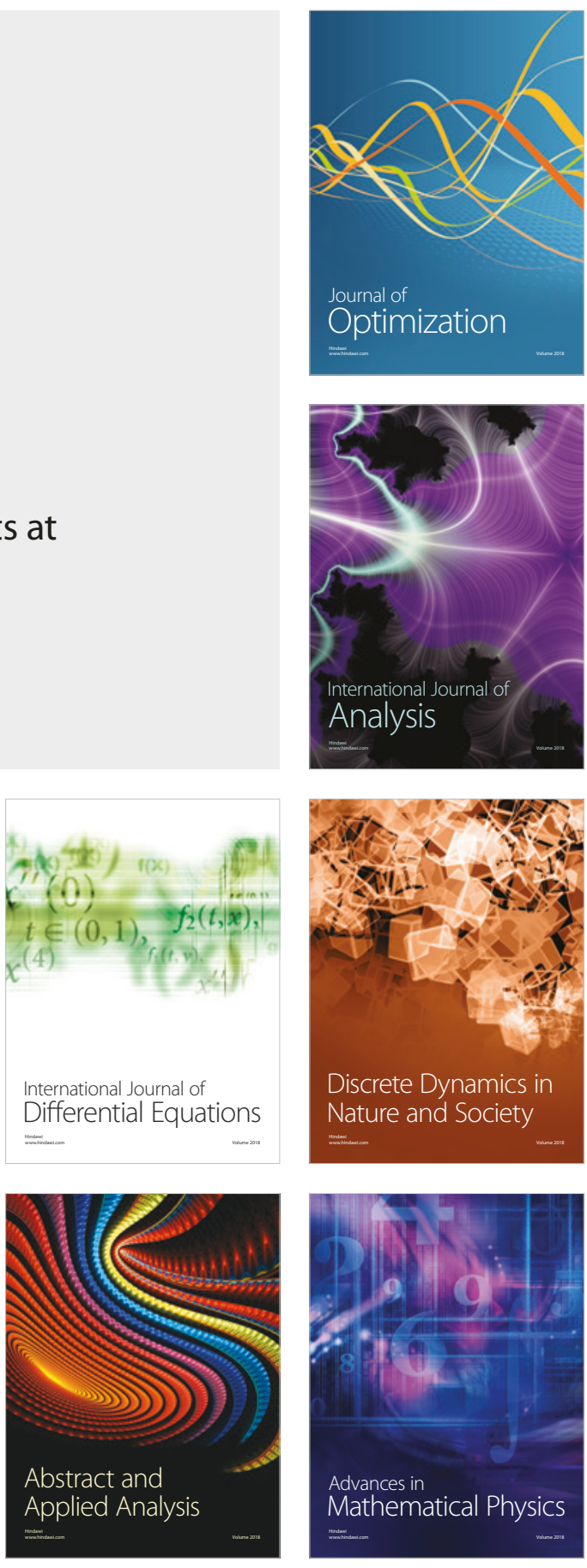